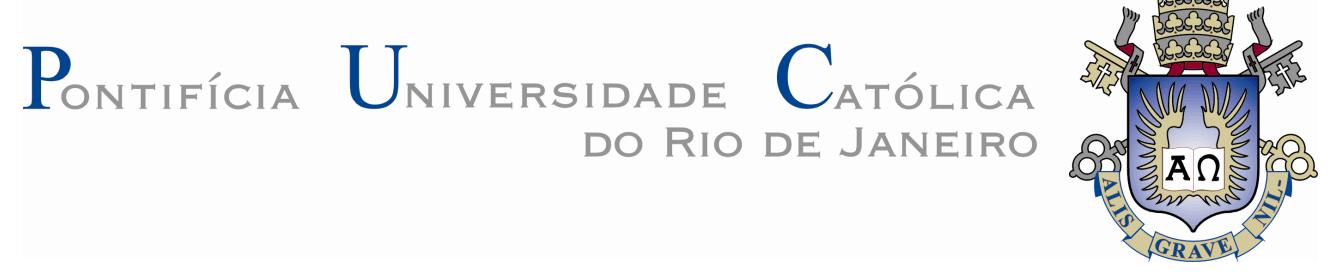

India Mara Martins

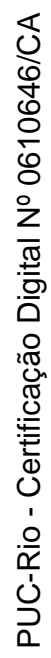

\title{
Documentário Animado: Experimentação, Tecnologia e Design
}

Tese apresentada ao Programa de Pós-graduação em Design da PUC-Rio como requisito parcial para obtenção do título de Doutora em Design.

Orientador: Prof. Luiz Antônio Luzio Coelho 


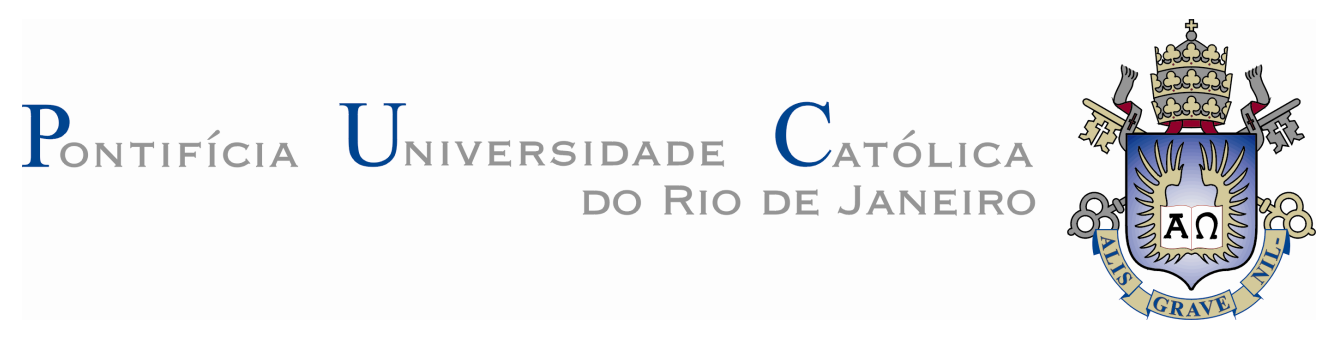

India Mara Martins

Documentário Animado:

Experimentação, Tecnologia e Design

Tese apresentada como requisito parcial para obtenção do grau de Doutor pelo Programa de Pós-Graduação em Design do Departamento de Artes e Design do Centro de Teologia e Ciências Humanas da PUC-Rio. Aprovada pela comissão examinadora abaixo assinada.

Prof. Luiz Antônio Luzio Coelho

Orientador

Departamento de Artes e Design - PUC-Rio

Prof. João Luiz Vieira

Departamento de Cinema e Audiovisual - UFF

Prof. Jorge Luiz Rocha de Vasconcellos

Departamento de Cinema e Filosofia - UGF

Profa. Denise Portinari

Departamento de Artes e Design - PUC-Rio

Prof. Newton Gamba Jr.

Departamento de Artes e Design - PUC-Rio

Prof. Paulo Fernando Carneiro de Andrade Coordenador Setorial do Centro de Teologia e Ciências Humanas - PUC-Rio

Rio de Janeiro, 08 de abril de 2009. 
Todos os direitos reservados. É proibida a reprodução total ou parcial do trabalho sem autorização da universidade, do autor e do orientador.

\section{India Mara Martins}

Graduada em Comunicação Social, pela Universidade Estadual de Ponta Grossa - UEPG, em 1992. Mestre em Multimeios pela Universidade Estadual de Campinas Unicamp. Trabalha como docente desde 1996 nas áreas de Cinema e Comunicação. De 2003 a 2006 coordenou a Produtora Modelo do Centro Universitário da Cidade - RJ, onde desenvolveu projetos de audiovisual como filmes interativos, animação e curtas-metragens.

Ficha Catalográfica

\begin{tabular}{|c|}
\hline Martins, India Mara \\
\hline $\begin{array}{l}\text { Documentário Animado: Experimentação, } \\
\text { Tecnologia e Design / India Mara Martins ; } \\
\text { orientador: Luiz Antônio Luzio Coelho. - } 2009 . \\
244 \text { f. : il. (color.) ; } 30 \mathrm{~cm}\end{array}$ \\
\hline $\begin{array}{l}\text { Tese (Doutorado em Artes e Design) - } \\
\text { Pontifícia Universidade Católica do Rio de Janeiro, } \\
\text { Rio de Janeiro, } 2009 \text {. } \\
\text { Inclui bibliografia }\end{array}$ \\
\hline $\begin{array}{l}\text { 1. Artes }- \text { Teses. } 2 . \text { Documentário } \\
\text { Animado. 3. Design. } 4 . \quad \text { Experimentação. } 5 . \\
\text { Tecnologia. 6. Teoria do cinema. I. Coelho, Luiz } \\
\text { Antônio Luzio. II. Pontifícia Universidade Católica do } \\
\text { Rio de Janeiro. Departamento de Artes e Design. III. } \\
\text { Título. }\end{array}$ \\
\hline
\end{tabular}

CDD: 700 
Este trabalho é dedicado ao Leon, meu filho querido, amigo e companheiro, que esteve presente em todos os momentos de sua realização e faz uma ótima massagem, apesar dos seus seis anos de idade. 


\section{Agradecimentos}

Diversas pessoas contribuíram direta ou indiretamente para a realização deste trabalho, na impossibilidade de citar todas, agradeço:

Ao meu orientador Luiz Antônio Luzio Coelho, pela confiança e pelos caminhos indicados ao longo deste percurso;

À Manuela Penafria, minha orientadora no Estágio de Doutorado na Universidade da Beira Interior, por sua generosidade intelectual e apoio;

À Mônica Moura, minha orientadora inicial, que por força das circunstâncias não acompanhou este trabalho até o fim, mas enquanto o fez, foi gentil e cuidadosa;

A todos os professores do Departamento de Artes e Design da PUC-Rio, especialmente à Denise Portinari pelas contribuições oferecidas a esta pesquisa e o apoio em todas as situações acadêmicas;

Aos animadores que concederam entrevista para a realização deste trabalho: Abi Feijó, Regina Pessoa, José Cavalheiro, o Zepe, César Cabral;

À amiga Mônica Carvalho pela amizade e apoio no Brasil e em Portugal.

Aos colegas das disciplinas pelos animados papos durante os intervalos: Guilherme Xavier, Ricardo Arthur, Fábio (Bola), Ana Paula, Izabel, Mônica Saddi, Tatiana, Kácia Diniz, André Ramos, Simone.

Agradeço especialmente ao amigo Daniel Risi pelas conversas sagazes e questionadoras e pelas referências em tecnologia. 
Daniel Pinna, amigo querido, merece um agradecimento especial pela sua presença na minha vida acadêmica, profissional e pessoal.

Às queridas amigas Mariane Koslinski por suas dicas acadêmicas e pela revisão das traduções, à Fernanda pelas conversas francesas, à Luciana pelo porto seguro em SP, à Rosane pelo apoio psicológico e espiritual e à Denise Assef, pelo ombro amigo e ajuda na formatação.

Agradeço a todos os colegas, que participaram do projeto Produtora Modelo, onde percebi a importância do design para o Audiovisual e optei por esta área de pesquisa: Robson, Sávio, Alexandre, Max, Gisele, Daniel.

A todos os animadores que gentilmente me concederam entrevistas: Abi Feijó, Regina Pessoa, José Cavalheiro (Zepe) e César Cabral, e aqueles que me inseriram no meio: Gabriel Cruz, Bruno Cruz, Pedro Iuá, Simone.

À Liana Koslinski que gentilmente recebeu a mim e ao meu filho em sua casa após o estágio de doutorado.

A Ana e Álvaro por sua amizade, confiança e apoio incondicional a todos os meus projetos.

A minha família, que mesmo distante, está sempre presente. 


\section{Agradecimentos Institucionais}

Agradeço à CAPES pelo financiamento do estágio de doutorado na Universidade da Beira Interior - UBI, em Covilhã, Portugal.

Ao Projeto Teoria e Estética do Documentário, coordenado por Manuela Penafria e financiado pela Fundação para Ciência e Tecnologia - FCT - Portugal pelo apoio financeiro para a participação em congressos nacionais e internacionais.

À FAPERJ - Fundação para Pesquisa do Rio de Janeiro, pelo apoio financeiro obtido através da Bolsa Nota 10 no último ano do doutorado.

À Pontifícia Universidade Católica do Rio de Janeiro - PUC-Rio pela bolsa positiva para a realização do doutorado.

À Universidade da Beira Interior - UBI, especialmente o Departamento de Cinema e seu coordenador Paulo Serra, por ter aberto seus laboratórios e bibliotecas para a realização da minha pesquisa.

À Cinemateca Portuguesa pelo acesso a artigos e livros mesmo no período das férias.

Ao British Institute de Coimbra pelo acesso aos filmes ingleses dos anos 30 . 


\section{Resumo}

Martins, India Mara; Coelho, Luiz Antônio Luzio (Orientador). Documentário Animado: Experimentação, Tecnologia e Design. Rio de Janeiro, 2009. 244p. Tese de Doutorado - Departamento de Artes \& Design, Pontifícia Universidade Católica do Rio de Janeiro.

O objetivo desta tese é refletir sobre o documentário animado. Um produto audiovisual que mistura documentário e animação e está redefinindo o papel do design na produção das novas mídias. Mostramos também que o documentário animado reacende uma série de debates e reflexões relativas à teoria do documentário e da animação em relação às concepções de realismo. A nossa principal premissa é que o documentário sempre se apropriou da tecnologia de forma a favorecer a experimentação e, o documentário animado, em 3D, é a sua forma de se apropriar das tecnologias digitais. Para comprovar esta hipótese mostramos como a experimentação da tecnologia se deu no documentário em diferentes épocas, mais precisamente nos períodos do primeiro cinema, das vanguardas e da escola britânica - de 1900 a 1930, os anos de 1960, e os anos de 1990 (novas mídias). O que nos interessa é investigar esta relação entre os meios de produção utilizados na realização do documentário - quer tenham esta denominação ou não - e os modos de representação e estilos resultantes da tecnologia de cada época, que pretendemos delinear brevemente. O Design está presente nos dois aspectos: nos meios de produção, por sua tradição ligada ao desenvolvimento de tecnologias e instrumentos para viabilizar a produção, e nos modos de representação, porque as referências estéticas da animação são oriundas das artes gráficas (quadrinhos, ilustrações, etc). Isto também explica, em parte, a ausência da animação na teoria do cinema, aspecto que questionamos, mostrando como a animação está contemplada na teoria Deleuziana. Para concluir, fazemos um estudo de caso de um documentário animado emblemático, por sua abordagem da tecnologia 3D. Ryan (2004), de Chris Landreth, resume o potencial do documentário animado enquanto experimentação da tecnologia, intensidade narrativa e estética não figurativa, em singular referência ao pintor Francis Bacon.

\section{Palavras-chave}

Documentário animado; design; experimentação; tecnologia; teoria do cinema. 


\section{Abstract}

Martins, India Mara; Coelho, Luiz Antônio Luzio (Advisor). Animated Documentary: Experimentation, Technology and Design. Rio de Janeiro, 2009. 244p. Dsc. Thesis - Departamento de Artes \& Design, Pontifícia Universidade Católica do Rio de Janeiro.

The objective of this thesis is to reflect on the animated documentary. An audiovisual product that mixes both documentary and animation and is redefining the role of the design in the production of new media. We also show that the animated documentary rekindled a series of debates and reflections related to documentary's theory and realism concepts in animation. Our main premise is that the documentary has always appropriated of technology in order to allow experimentation, and the animated documentary, in 3D, is the way to appropriate itself of digital technologies. To prove this hypothesis, we show how the trial of the technology occurred in documentary's history at different times, more precisely during the periods of the early cinema, the vanguards and the British School - from 1900 to 1930, the 1960's and the 1990's (new media). What concern us is to investigate this relationship between the means of production used in the making of documentary movies - having this denomination or not - and ways of representation and styles resulting from the technology of each period, which we intend to outline briefly. The Design is present in both two aspects: in the means of production - for its tradition related to the development of technologies and tools to make production viable - and in the ways of representation, since the aesthetic references of animation cinema came from the graphic arts (comics, illustration, etc.). This also explains, partially, the absence of animation in cinema's theory, an aspect that we question, showing how animation is included in Deleuze's cinema's theory. Finally, we develop a case study of an emblematic animated documentary, for its approach to the 3D technology. Chris Landreth's Ryan (2004) is a synthesis of potential of the animated documentary as technological experimentation, narrative intensity and non-figurative aesthetic, in a unique reference to the painter Francis Bacon.

\section{Keywords}

Animated Documentary; Design; Experimentation; Technology; Theory of Cinema. 


\section{Resumé}

Martins, India Mara; Coelho, Luiz Antônio Luzio (directeur). Documentaire Animé: Experimentation, Technologie et Design. Rio de Janeiro, 2009. 244p. Thèse de Doctorat - Departamento de Artes \& Design, Pontifícia Universidade Católica do Rio de Janeiro.

Cette thèse a le but de réfléchir sur le documentaire animé. Un produit audiovisuel qui mélange documentaire et animation et qui est en train de redéfinir le rôle du design dans la production des nouveaux médias. Nous montrons que le documentaire animé rallume une série de débats et de réflexions sur la théorie du documentaire et de l'animation concernant les conceptions du réalisme. Notre principale prémisse est que le documentaire s'est toujours approprié de la technologie de manière à favoriser l'expérimentation, et le documentaire animé en 3D est la façon dont il s'approprie des technologies numériques. Pour prouver cette hypothèse, nous montrons comment s'est déroulée l'expérimentation de la technologie dans le documentaire dans de différentes époques, plus précisément durant les périodes du premier cinema, les avant-gardes et l'école britannique -de 1900 à 1930, les années 1960 et 1990 (nouveaux médias). Ce qui nous intéresse c'est d'étudier la relation entre les moyens de production utilisés dans la réalisation du documentaire -que ce soit avec cette dénomination ou pas- et les manières de représentation et de styles résultant de la technologie de chaque période, que nous prétendons ébaucher brièvement. Le design est présent dans deux aspects: dans les moyens de production, par sa tradition liée au développement de technologies et d'outils pour faciliter la production, et dans les manières de représentation, parce que les références esthétiques de l'animation sont des arts graphiques (bandes dessinées, illustrations etc.). Ceci aussi explique, en partie, l'absence de l'animation dans la théorie du cinéma, un aspect que nous questionons, en montrant comment l'animation est envisagée dans la théorie deleuzienne. Pour conclure, nous faisons une étude de cas d'un documentaire animé emblématique, par son traitement de la technologie 3D. Ryan (2004), de Chris Landreth, résume le potentiel du documentaire animé tout en expérimentant avec la technologie, l'intensité narrative et l'esthétique non figurative, en singulière référence au peintre Francis Bacon.

\section{Mots-Clé}

Documentaire Animé ; Design; Experimentation; Technologie ; Théorie du Cinéma. 


\section{Sumário}

1. Introdução Geral

Parte 1 Documentário, Tecnologia e Experimentação

26

2. O documentário e o Primeiro Cinema

26

2.1. Breve Descrição do Primeiro Cinema

26

2.2. Alguns Conceitos sobre o Primeiro Cinema

28

2.3. As Atualidades e a Câmera em Movimento

32

2.4. Registros da realidade

36

3. O documentário e as Vanguardas dos anos 20

49

3.1. Aspectos formais e condições de produção 55

3.2. Os documentários de vanguarda 59

$\begin{array}{ll}\text { 3.3 . Filmes sinfonia e cidades imagéticas } & 67\end{array}$

4. Escola Britânica: a definição de documentário nos anos 30

4.1. A introdução do som no documentário 72

4.2. A definição do cinema de não-ficção 76

4.3. Aspectos formais da escola britânica $\quad 87$

$\begin{array}{ll}\text { 4.3.1. Drifters } & 87\end{array}$

$\begin{array}{ll}\text { 4.3.2. Song of Ceylon } & 91\end{array}$

4.3.4. Reflexões pós Grierson sobre o cinema documentário 94

5. Os cinemas novos e a tecnologia liberam o documentário 98

5. Cinéma Verité 100

5.2. Cinema Direto americano 102

5.3. Algumas críticas 104

5.4. Anos 90 e as imagens de síntese 105 
6.1. Princípios da Animação 117

6.2. Questões sobre o realismo de Disney 121

6.2.1. O estilo UPA e a ruptura com o realismo Disney 123

6.2.2. O Estilo Japonês - inspirado no realismo de Disney 126

6.2.3. A questão autoral na animação 127

6.2.4. Nos EUA 132

6.2.5. O Estilo Escola de Zagreb 133

6.2.6. O estilo experimental do NFB 134

6.3. Conceitos de realismo no cinema live-action 137

6.3.1. O realismo e a tecnologia 142

6.3.2. Realismo Perceptual 146

$\begin{array}{lr}\text { 6.3.3. Opacidade e transparência } & 149\end{array}$

7. O Documentário Animado 152

7.1. O campo teórico do documentário e o documentário animado 154

7.2. Definindo documentário animado 159

$\begin{array}{ll}\text { 7.2.1. Tipos de documentário animado } & 160\end{array}$

7.2.2. Funções retóricas da animação 162

7.2.3. Função ideológica da animação nos filmes instrucionais 165

8. O Documentário Animado e o Design 168

8.1. Estudo de Caso - Ryan (2004), de Chris Landreth 172

8.1.1. Histórico do Projeto 172

$\begin{array}{ll}\text { 8.1.2. Estratégias Narrativas } & 174\end{array}$

$\begin{array}{ll}\text { 8.1.3. A estrutura narrativa de Ryan } & 178\end{array}$

8.1.4. Etapas na produção de uma animação 181

8.1.5. Aspectos estéticos: técnicas e ferramentas 190

8.1.6. Atmosfera onírica 194 
9. O Documentário Ryan e o Psicorrealismo

9.1. Francis Bacon por Deleuze 200

$\begin{array}{ll}\text { 9.2. A figura } & 201\end{array}$

9.2.1. Cabeças e rostos 205

9.2.2. Pintar a sensação 207

9.2.3. Pintar forças, animar sensações 209

9.2.4. Tornar visíveis as forças invisíveis $\quad 212$

10. Conclusão Resumo 215

11. Referências Bibliográficas 222

$\begin{array}{ll}\text { Sites Citados } & 229\end{array}$

$\begin{array}{ll}\text { Filmografia Chris Landreth } & 231\end{array}$

Filmografia Documentário animado $\quad 231$

Filmografia Primeiro Cinema 232

Filmografia Vanguardas $\quad 233$

Filmografia Documentários $\quad 233$

Filmografia Animação $\quad 235$

$\begin{array}{ll}\text { Filmografia Cinema em geral } & 238\end{array}$

Anexo Decupagem Documentário Animado Ryan 239 


\section{Índice de llustrações}

1.i Rough Sea at Dover (1895) Birt Acres 29

1.ii L'arrivée d'un train em gare de la Ciotat (1895) Lumière 38

1.iii Sky scrapers of New York City from North River, de 1903, da Cia. de Edison 42

1.iv San Francisco: Afterrath of Earthquake, de 1906, de Thomas Edison 43

1.v Moscow clad in snow - de Pathé Fréres, de 1908

2.i The Sinking of the Lusitânia (Winsor McCay, 1918, US) 49

2.ii Le Retour à la Raison (1923), Man Ray 51

2.iii La Coquille et le Clerygman (1929),Dulac 56

2.i Ballet Mécanique (1925) 60

2.v Chuva (1929), de Joris Ivens 61

2.vi Kino-Eye (1924), de Dziga Vertov 63

2.vii Kino-Eye (1924), de Dziga Vertov 65

3.i Rien que les Heures (1929), Alberto Cavalcanti 78

3.ii Trade Tattoo (1937), Len Lye 83

3.iii Drifters (1929), John Grierson 88

3.iv Song of Ceylon (1934), Basil Wright, 92

4.i Moi, um noir (1958), Jean Rouch 100

4.ii Primary (1960), Robert Drew 104

4.iii Mr. Death (1999), Errol Morris 106

5.i Blackton e seu lightning sketch Humorous Phases of Funny Faces (1875) 110

5.ii Primeiro mecanismo de Rotoscopia patenteado por Max Fleischer em $1917 \quad 113$

5.iii Primeiro mecanismo de Rotoscopia patenteado por Max Fleischer em $1917 \quad 114$

5.iv Estudos para Branca de Neve e os Sete Anões (1938), Disney 115

5.v Animador dos estúdios Disney observando um cervo. 116

5.vi Oliver Martin Johnston Jr. 116

5.vii Storyboard Mr. Magoo, John Hubley 124

5.viii A Turma do Manda Chuva, Hanna Barbera 125

5.ix O estilo fluído de "banda desenhada" de Stuart 129

5.x A Night on Bald Mountain (1933) 131 
5.xi Alexandre Aleixeïeff no NFB 131

5.xii American Pop (1981), Ralph Bakshi 133

5.xiii Superposição de imagens em Pas de deux (1967) 136

5.xiv Matrix (2001), irmãos Wachowski 142

5.xv Walking with Dinosaurs (1999) 143

5.xvi Animated Minds, Andy Glynne, 2003.

5.xvii Abductees (1995), de Paul Vester 150

6.i The sinkhing 152

6.ii Hell Unltd. 152

6.iii Hell Unltd. 152

6.iv Trade Tattoo 152

6.v. Chicago 10

6.vi llustrações de You don't have to die, de 1988.

6.ii Victory Through Air Power C.Geronimi, J.Kinney, J.Alger e H.C. Potter, 1943165

$\begin{array}{ll}\text { 7.i Mr. Death } & 171\end{array}$

7.ii Bingo (1998), Chris Landreth 173

7.iii Seqüência Ryan: 1 a, 1c e 1d 178

7.v Seqüência $2 \mathrm{~g}$, 2h e $2 \mathrm{r} \quad 179$

7.vi Seqüência 3j, 4d e 4l 180

7.vii Seqüência 8o, 8q e 9c 180

$\begin{array}{ll}\text { 7.viii Seqüência 10d, 10e e 10i } & 181\end{array}$

7.ix Esquerda para direita: a) Foto de Ryan Larkin. b) A modelagem de Larkin pela 184

CG. c) As impressões de Landreth sobre Ryan, desenhadas durante a entrevista. 184

d) A caracterização final de Ryan, criada com carvão e modelada pela CG. 184

7.x Seqüência 1.L, 1c e 1p 184

7.xi Seqüência $5 a, 5 b$ e 5n 185

7.xii Seqüência 2p, 2q e 2r 188

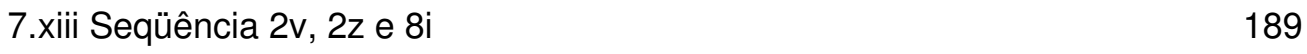

7.xiv Seqüência 5o, $5 p$ e $6 a \quad 193$

7.xv Seqüência 7c, 7d e 7h 193

7.xvi Seqüência 8L, 8z e 9o 194

7.xvii Boulevard de Montreal recriado em 3D 195

7.xviii Perspectiva não linear da cafeteria do abrigo 196

7. xix Banheiro distorcido pela perspectiva não linear 197

8.i Francis Bacon Triptico (1974-1977) 201

8.ii Seqüência 4i, 7e e 8i 203

8. iii Seqüência 1c, 1f e 1n 204 
8. iv Seqüências 9e, 9f e 9j 205

8. v Francis Bacon, Três Estudos para Auto-retrato (1976) 206

8.vi Seqüências 2i, 2n e 2r 206

8.vii Seqüências 2g, 3f e 4n 206

8. viii Lembrança da mãe de Chris - O desequilibra 209

8. ix Seqüências 1c. 1d e 1e 210

8. x Seqüências $2 g$, $8 d$ e $8 g \quad 210$

8. xi Seqüências $5 b, 5 c$ e $5 i \quad 211$

8. xii Seqüências $6 f, 6 \mathrm{~g}$ e $6 \mathrm{~h} \quad 212$

8. xiii Seqüências 7c, 7d e 7e 212 